Port Econ J (2014) 13:95-115

DOI 10.1007/s10258-014-0099-1

ORIGINAL ARTICLE

\title{
The effect of education, family size, unemployment and childcare availability on birth stopping and timing
}

\author{
Márton Varga
}

Received: 14 March 2012 / Accepted: 9 May 2014 / Published online: 28 June 2014

(C) ISEG 2014

\begin{abstract}
Using data from Portugal's Fertility and Family Survey, I analyze childbearing decisions up to the third birth using a split-population (SP) model. The advantage of this approach is the separability of the covariates' impact on birth timing and birth stopping. This paper is the first to apply an SP model to investigate the effect of unemployment and the availability of childcare. I also address how education, family size, age at previous birth of the woman and sex composition of existing children influence childbearing decisions, and provide empirical support for each of these. Comparing these with estimates obtained using survival models that do not include a regression on birth stopping suggests that the results of the latter tend to be unreasonable.
\end{abstract}

Keywords Fertility $\cdot$ Split population model $\cdot$ Event history analysis · Parity progression $\cdot$ Birth timing $\cdot$ Portugal

JEL Classifications C41 $\cdot$ J13 $\cdot$ D10 